\title{
Noninvasive harmonics optical microscopy for long-term observation of embryonic nervous system development in vivo
}

\author{
Szu-Yu Chen \\ Cho-Shuen Hsieh \\ Shi-Wei Chu \\ National Taiwan University \\ Department of Electrical Engineering and \\ Graduate Institute of Electro-Optical Engineering \\ Taipei 10617, Taiwan
}

\section{Cheng-Yung Lin}

National Taiwan University Institute of Molecular and Cellular Biology Taipei 10617, Taiwan

\section{Ching-Yi Ko \\ Yi-Chung Chen}

National Taiwan Ocean University

Institute of Bioscience and Biotechnology

Keelung 20224, Taiwan

\section{Huai-Jen Tsai}

National Taiwan University

Institute of Molecular and Cellular Biology

Taipei 10617, Taiwan

\section{Chin-Hwa Hu}

National Taiwan Ocean University Institute of Bioscience and Biotechnology Keelung 20224, Taiwan

\author{
Chi-Kuang Sun \\ National Taiwan University \\ Department of Electrical Engineering and \\ Graduate Institute of Electro-Optical Engineering \\ Taipei 10617, Taiwan
}

\begin{abstract}
Nervous system development is a complicated dynamic process, and many mechanisms remain unknown. By utilizing endogenous second-harmonic-generation as the contrast of polarized nerve fibers and third-harmonic-generation (THG) to reveal morphological changes, we have successfully observed the vertebrate embryonic nervous development from the very beginning based on a 1230-nm light source. The dynamic development of the nerve system within a live zebrafish embryo can be recorded continuously more than $20 \mathrm{hr}$ without fluorescence markers. Since the THG process is not limited by the time of gene expression and differentiation as fluorescence-based techniques are, the observable stages can be advanced to the very beginning of the development process. The complete threedimensional brain development from a neural plate to a neural tube can be uncovered with a submicron lateral resolution. We have, for the first time, also reported the generation of SHG from myelinated nerve fibers and the outer segment of the photoreceptors with a stacked membrane structure. Our study clearly indicates the fact that higher-harmonics-based optical microscopy has the strong potential to long-term in vivo study of the nervous system, including genetic disorders of the nervous system, axon pathfinding, neural regeneration, neural repair, and neural stem cell development. ๑ 2006 Society of Photo-Optical Instrumentation Engineers. [DOI: 10.1117/1.2363369]
\end{abstract}

Keywords: harmonics; optical microscopy; noninvasive; nervous system; in vivo; polarized nerve fibers.

Paper 05312R received Oct. 18, 2005; revised manuscript received Mar. 20, 2006; accepted for publication May 5, 2006; published online Oct. 30, 2006.

\section{Introduction}

Optical higher-harmonic generations, including secondharmonic-generation (SHG) and third-harmonic-generation (THG) processes, are known to leave no energy deposition to the interacted matters due to the virtual-transition characteristic. ${ }^{1}$ In contrast to the absorption-inducedfluorescence processes that require energy deposition and electron transitions, the higher-harmonic generation processes provide the optical noninvasive nature desired for microscopy applications, especially for long-term observation of the dynamic changes of live samples. Different from single-photon

Address all correspondence to: Chi-Kuang Sun, National Taiwan University, Department of Electrical Engineering and Graduate Institute of Electro-Optical Engineering, Taipei 10617, Taiwan. Tel: +886-2-3366-5085; Fax: +886-2-33663614; E-mail: sun@cc.ee.ntu.edu.tw and multiphoton fluorescence, no cell damage and photobleaching effect is expected from the optical harmonicgeneration process itself due to the fact that there is no real electron transition involved and the total generated harmonic photon energy has to be equal to the total annihilated photon energy. ${ }^{2}$ With a nonlinear nature similar to the multiphoton excited fluorescence, the generated SHG intensity depends on the square of the incident light intensity, while the generated THG intensity depends on the third power of the incident light intensity. These nonlinear dependencies allow localized excitations to enable intrinsic optical sectioning and a high threedimensional resolution similar or better than that of the twophoton fluorescence microscopy. ${ }^{3,4}$ THG is known to be a fundamental physical process during which the THG intensity

1083-3668/2006/11(5)/054022/8/\$22.00 @ 2006 SPIE 
peaks when the foci of the strongly focused optical beams are located at the material interfaces. ${ }^{5-8}$ In biological tissues, THG arising from interfaces including various membranes and organelles, especially mitochondria, ${ }^{9-12}$ can be used to reveal the morphological structures. ${ }^{13,14}$ On the other hand, SHG contrast in biological specimens can be provided by ordered arrangement of highly asymmetric biomolecules where the optical centrosymmetry is broken, including stacked membranes ${ }^{15,16}$ and structured proteins. ${ }^{17-23}$ Combined with a least-invasive 1230-nm light source, higher-harmonic generation microscopy has been proven to be an excellent tool for studying vertebrate embryonic development in previous research. $^{13,14}$

Among many vertebrate physiological systems, the nervous system is one of the most important and complicated systems, and its embryonic development process plays a significant role and is worth our efforts to solve the puzzles remaining. Since it takes charge of regulating the actions and reactions of the whole body, any disorders occurring in the developing nervous system may lead to serious diseases. It is thus important to understand how the nervous system is developed and how it functions. In order to find the diseasecausing genes and how they affect the nervous system, several genetic models, such as Dorsophila (the fruit fly), and zebrafish (Danio rerio), have been adopted, especially the zebrafish. Because the zebrafish has much genetic material that is the same as humans, scientists can learn how the genes function in the human body by studying zebrafish. Compared with humans, the zebrafish has similar but simpler developmental programs during early embryonic stages. Thus, the zebrafish has become a standard vertebrate model, and different phenotypes of zebrafish have been developed for investigating the gene functions in the nervous system. ${ }^{24}$ In addition, its rapid developing rate, precisely defined developing stages, transparency, small size, and external development also facilitate the neurology study and microscopic observation.

Although development of the nervous system is a dynamic process, static images obtained from cryostat sectioning of different embryos are often used during study. Recently, advances in live imaging systems, such as ultrasound imaging, ${ }^{25}$ magnetic resonance imaging, ${ }^{26,27}$ optical coherence tomography, ${ }^{28,29}$ confocal fluorescence microscopy, ${ }^{30,31}$ and multiphoton fluorescence microscopy, ${ }^{32-34}$ have offered many opportunities to provide insights into the development process. Besides, other functional research, such as $\mathrm{Ca}^{2+}$ imaging $^{35,36}$ and fluorescence resonance energy transfer, ${ }^{37}$ were also used to reveal how the nervous system works. While nonfluorescence-based techniques are limited by the spatial resolution, the widely used fluorescence-based techniques are restricted to the observable periods of the development process and by the applied excitation power. Since most fluorescence signals are controlled by the differentiation process due to neuron-specific promoters, the earliest observable stage of development will be restricted to when the fluorescence is expressed. To the best of the authors' knowledge, long-term in vivo and noninvasive observations of the complete neural development process have never been successfully achieved in a live vertebrate embryo, while different part and stages of the early nervous development can be observed with a submicron resolution. Moreover, since a fluorescencebased technique always accompanies photo-energy deposi- (a)

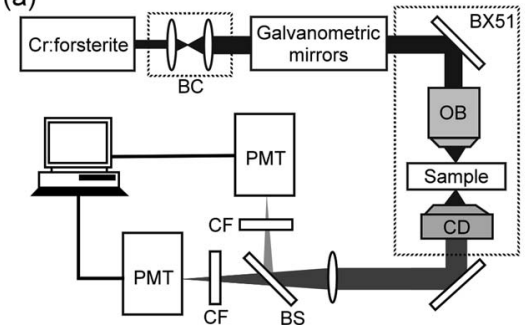

(b)

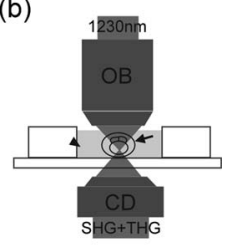

Fig. 1 (a) The scheme of the laser-scanning higher-harmonic optical microscope (HOM) system. The system was adapted from an Olympus FV300 scanning unit combined with an Olympus BX51 upright microscope. $\mathrm{BC}$, beam collimator; $\mathrm{OB}$, objective; $\mathrm{CD}$, condenser; $\mathrm{BS}$, beam splitter; CF, color filter; PMT, photomultiplier tube. (b) The scheme of the miniaturized aquarium, where the live zebrafish embryo (arrow) without any treatment was kept in water (arrowhead).

tion, and photodamage and photobleaching effects always raise discussion, ${ }^{38,39}$ the power after objective has to be under a threshold value, thus limiting the maximum penetration depth for in vivo observation.

In this paper, for the first time, the whole vertebrate embryonic nervous development is successfully observed in vivo from the very beginning. Higher-harmonics-generation is applied to continuously observe the dynamic development of the nervous system within a live zebrafish embryo for more than $20 \mathrm{hr}$. The in vivo study was performed with a 1230-nm light source, thus providing high cell viability ${ }^{14}$ and allowing THG to fall within the visible spectrum for deep-tissue observation. Endogenous SHG is utilized as the contrast of nerve fibers ${ }^{40,41}$ and mitotic spindles, ${ }^{14,19}$ while THG is used to reveal the cell behaviors and morphological changes. Since the THG process is not limited by the time of gene expression and differentiation as fluorescence-based techniques are, the observable stages can be advanced to the very beginning of the whole development process. The complete three-dimensional brain development from a neural plate to a neural tube can be uncovered with a submicron lateral resolution. Since no fluorophores and live markers are needed, toxicity, photobleaching, and other biological as well as genetic environment modifications can all be completely avoided. After observing the brain development of several tens of zebrafish embryos, with nonstop continuous observation time in a single embryo even up to $20 \mathrm{hr}$, the embryos can all develop normally into the larva stages. Our study indicates that higher-harmonics-based optical microscopy has the strong potential to long-term in vivo study of the nervous system, including genetic disorders of the nervous system, axon pathfinding, neural regeneration, neural repair, and neural stem cell development.

\section{Method}

The laser-scanning, higher-harmonic optical microscope (HOM) system [Fig. 1(a)] was adapted from an Olympus FV300 scanning unit combined with an Olympus BX51 upright microscope, and all optics were modified to allow the passage of the 1200-1350-nm infrared light. The light source was a home-built $\mathrm{Cr}$ :forsterite laser, functioning at $1230 \mathrm{~nm}$ with a repetition rate of $110 \mathrm{MHz}$ and a pulse width of 140 fs. Real-time 2D scanning was accomplished with a pair 
of high-speed galvanometer mirrors inside the scanning unit. The collimated laser beam was coupled into the scanning system connecting to an Olympus BX51 microscope with an aperture-fitting tube lens. In order to visualize the whole brain structures inside an untreated live zebrafish embryo whose diameter was $1.5 \mathrm{~mm}$ thick with chorion, the working distance of the objective had to be longer than $1.5 \mathrm{~mm}$. Through a 2-mm-working-distance high numerical-aperture (NA) infrared objective (LUMPlanF1/IR 60X/water/NA0.9, Olympus), the excitation laser pulse was focused into the desired location inside the specimen and scanning with a spot size close to its diffraction limit. The average power after the objective was $\sim 100 \mathrm{~mW}$. With a finite sample thickness, instead of collecting the signals in the backward direction, ${ }^{42}$ we collected the forward-propagating optical harmonics signals with a high-NA (1.4) oil-immersion condenser. The signals were guided into two photomultiplier tubes (PMT), which were synchronized with the galvanometer mirrors and used to respectively record the interference-filtered SHG and THG signals point by point to form $2 \mathrm{D}$ sectioned images. Additionally, stereographic 3D images of the whole live embryo can be obtained by controlling the depth of focus inside the specimens, and the movement of the live embryos can be observed by continuously scanning on the same specimens with a recording speed of $0.25-2.5 \mathrm{sec}$ per frame, with $512 \times 512$ scanning points. During observation, the live zebrafish embryos with their chorion were kept inside a miniaturized aquarium [Fig. 1(b)] without any treatment at room temperature $\left(20 \pm 1^{\circ} \mathrm{C}\right)$. In order to prevent the observation from being interfered with by the pigments, embryos were continuously treated with $0.003 \%$ PTU (Phenylthiourea) from 12-hr post-fertilization (hpf). PTU, a well-known technique, is now widely used in the developmental study of zebrafish to suppress pigmentation in embryos. ${ }^{43}$ The harmonics signals collected are represented by two different pseudocolors, green for SHG and blue for THG.

In our previous report, in live zebrafish embryos with the same objective, the spatial resolutions for the THG and SHG modalities inside the embryo had been experimentally measured to be $400-500 \mathrm{~nm}$ and $500-600 \mathrm{~nm}$ in lateral ${ }^{13}$ and on the order of $1 \mu \mathrm{m}$ in axial. For the work presented in this paper, we have successfully performed continuous observation for $20 \mathrm{hr}$ in each live embryo, and the observed results have been repeated more than 10 times (only one of those is shown in this article). Comparing our results with the previous time-lapse studies, no observable differences, including morphological changes and developing rate, were found within the observed period of embryonic development. That is to say that no bio-damage was observed while cell viability and cloning rate were both successfully preserved. ${ }^{38}$

\section{Results and Discussion}

\subsection{Long-Term and in vivo Observation of the Brain Development}

Without any fluorescence markers, we successfully accomplished nonstop, long-term observation of the brain development in the same live zebrafish embryo for more than $20 \mathrm{hr}$. The morphogenetic changes of the brain can all be picked up by the THG modality to show the development process from neural plate to neural keel, neural rod, and then neural tube.
On the other hand, the dynamics of the cell mitosis, neuron differentiation, and the nerve fibers in the brain can be recorded by the SHG modality.

According to the developing process described by C. B. Kimmel, ${ }^{44}$ the brain development of a zebrafish embryo begins at the bud stage, which is the end of the patterning phase. The patterning phase is an important phase of the vertebrate embryonic development, which is considered worthwhile to focus on, since many genes begin to be promoted and expressed. Using our home-built HOM system, we could observe the developing process from the one-cell stage, to the very beginning of the process, to 2 -days' larva in vivo. ${ }^{14} \mathrm{How}-$ ever, because the brain begins to develop at the bud stage, only the developing process after that and several significant stages during brain development will be discussed in this article.

To start the observation, a live bud-stage embryo was put into the miniaturized aquarium under the microscope. With the animal pole of the embryo upward and by scanning the laser beam around the animal pole, a cluster of cells was revealed by the sectioned $2 \mathrm{D}$ image through the THG modality [blue part in Fig. 2(a)]. Since some cells in this cluster were still undifferentiated and were able to divide, by monitoring this cluster of cells continuously, we could find spindles of mitosis in undifferentiated cells through strong SHG signals [green part in Fig. 2(a)]. When we moved the focus to different depths to optically section the $3 \mathrm{D}$ structure of this cluster, a platelike 3D structure could be found. Compared with the results of previous time-lapse studies, ${ }^{44}$ this structure can be easily identified to be the neural plate, the primordium of the central nervous system at the bud stage. Vigorous cell division in the neural plate was revealed by SHG through the observation of mitosis spindles.

The monitoring kept going for about $1 \mathrm{hr}$, and the embryo was then at the 3 -somite stage. From THG images, the neural plate could be found to be thickened and a middle line [arrow in Fig. 2(b)] was visible. During brain development, this change indicates that the neural plate is being epithelial infolded at the middle line to become a neural keel, another transient structure. However, because the rate of cell mitosis at this stage is retarded and no nerve fibers exist, almost no SHG signals were observed inside the neural keel. As the embryo developed into the 5-somite stage, SHG signals appeared again in the middle of the neural keel [green part in Fig. 2(c)]. Gradually, they became stronger and extended to be almost linear. We saw that the first neuron inside the neural keel began to differentiate at the 5-somite stage and elongated the nerve fibers, ${ }^{44}$ which generated the SHG signals and allowed us to observe noninvasively.

As the development went on, the morphological changes resulting from epithelial folding could all be recorded by the THG modality. By sectioning the transient structure at different depths and reconstructing the obtained THG images, a rodlike solid structure, called a neural rod, could be identified at about the 14-somite stage. From a single section of the rodlike structure [Fig. 2(d)], we found that the SHG signals arising from the nerve fibers in the neural rod were much stronger and became even more linear-like. After this stage, when the embryo developed into the 18-somite stage, several small cavities were observed to form in the rod. This process is called cavitation, which changes the rod to a hollow struc- 

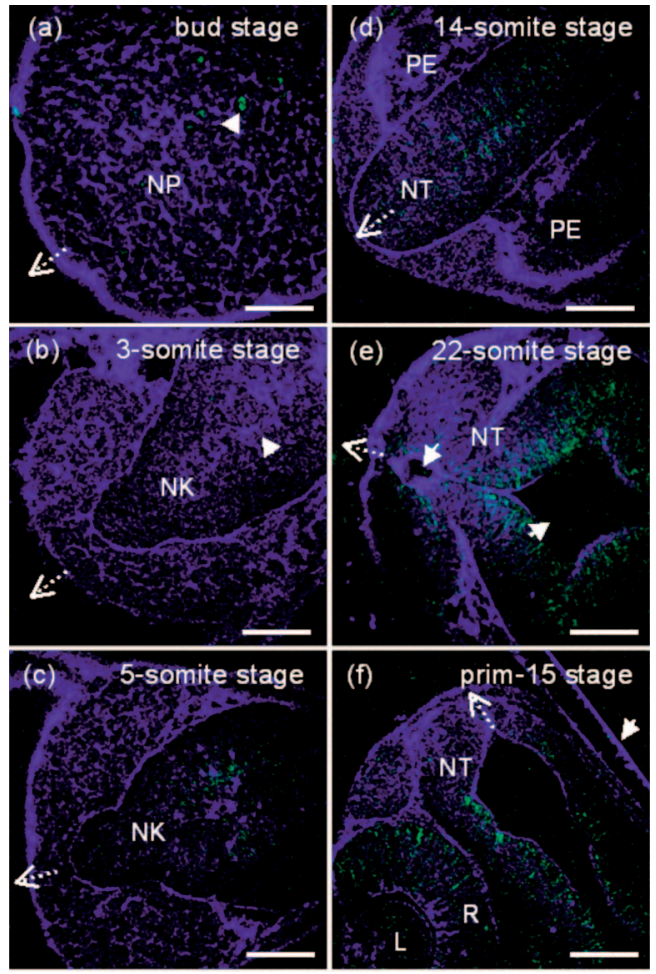

Fig. 2 Long-term in vivo observation of the brain development in the same zebrafish embryo from the bud stage to the prim-15 stage. The embryo was settled with the dorsal side to the top, and the dotted arrow shows the posterior-to-anterior direction. The HOM images shown here are all optically sectioned 2D images. (a) The neural plate (NP) at the bud stage. Vigorous cell mitosis at this stage was revealed by SHG (arrow). (b) The neural keel (NK) at the 3-somite stage. From THG, the middle line (arrow) where neural plate infolded to form the neural keel is visible. Since mitosis was retarded and no nerve fibers existing yet, almost no SHG was picked up. (c) The neural keel (NK) at the 5-somite stage. The SHG from nerve fibers was already observable near the middle line. (d) The neural tube (NT) at the 14-somite stage. The neural rod was formed and SHG became stronger and linear-like. This image also shows the primordium of the eyes (PE) by THG. (e) The midbrain at the 22-somite stage. Lumens (arrow) surrounded by neural tubes (NT) became more distinct. (f) The neural tube (NT) and the eye at the prim-15 stage. The lens (L) and retina (R) became distinguishable through THG, while the nerve fibers both in the neural tube and in the retina were revealed by SHG. Since the chorion of the embryo was not removed, it was shown by THG (arrow). Scale bar: $50 \mu \mathrm{m}$. THG and SHG are denoted by blue and green colors, respectively.

ture and forms the neural tube. ${ }^{44}$ From then on, the lumens surrounded by the neurocoele in the midbrain can be found to be more and more distinguishable, and they were clearly observed through THG signals when the embryo was at the 22somite stage [Fig. 2(e)]. Passing through the segmentation period, the lumens of the brain almost took shape at the prim-15 stage [shown by THG in Fig. 2(f)]; and THG also showed the structure of the eyes, including the retina and the lens [Fig. 2(f)]. In addition to the nerve fibers in the neural tube, the axons elongated from the ganglia in the retina were also observed through the SHG. Moreover, since the chorion was not removed, we could see the chorion around the developing embryo from the THG signals [arrow in Fig. 2(f)].
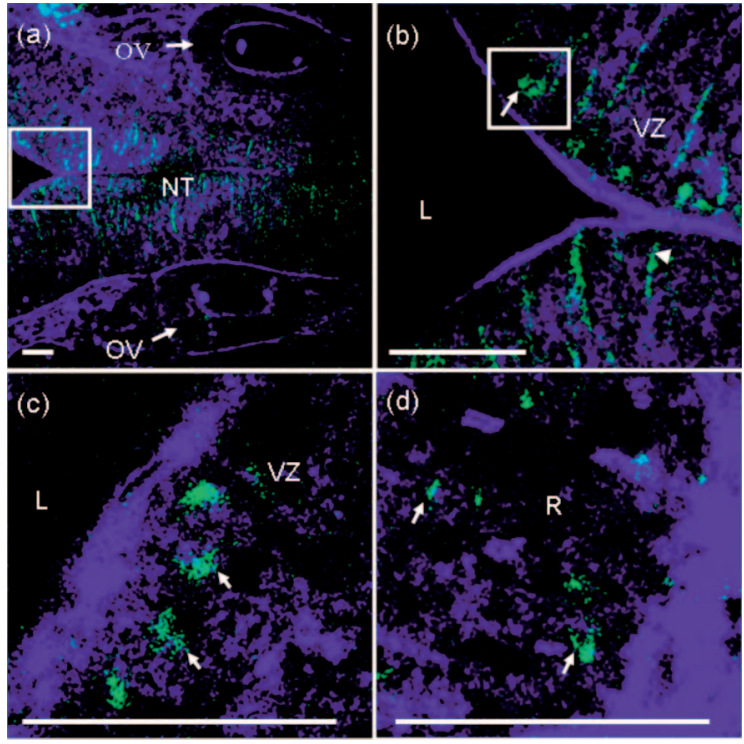

Fig. 3 SHG signals in the nervous system. (a) THG shows the outline of the neural tube (NT) and optic vesicles (OV) of a live prim-12-stage zebrafish embryo; and SHG reveals the nerve fiber distribution in the neural tube. (b) Inset from (a) shows the SHG in the ventricular zone (VZ) near the lumens (L). In this region, SHG arises from both mitotic spindles (arrow) and nerve fibers (arrowhead). (c) Enlarged image corresponding to the area of inset in (b) clearly shows the mitotic spindles (SHG; arrow) in the ventricular zone of the neural tube. (d) Cell mitosis (arrow) at the bottom of the retina (R). Scale bar: $20 \mu \mathrm{m}$. THG and SHG are denoted by blue and green colors, respectively.

Due to the least-invasive nature of HOM, the important morphogenetic changes of the zebrafish brain from the bud stage to the prim-15-stage were monitored in vivo continuously in the same live embryo without any fluorescence markers. Through the THG modality, we were able to record not only the 3D change of shape within several different transient structures, but also the dynamic cell behaviors, such as its movement and division. On the other hand, cell mitosis, cell differentiation, and nerve fiber developments in the brain and the retina can all be observed from SHG signals. Although some observations of nervous system development have been demonstrated using fluorescence-based confocal microscopy, ${ }^{45}$ the earliest observable stage was limited by the time at which the fluorescence protein was expressed in the neuron cells. Compared with the earliest stage-the prim-15 stage of a zebrafish embryo-when confocal fluorescence microscopy can be used to observe, the HOM system can advance the observation time to at least $14 \mathrm{hr}$ sooner. Not only the behavior of differentiated neuron cells but also the undifferentiated cells can all be recorded to provide a complete 4-dimensional (3 spatial dimensions and time) record of nervous system development. Moreover, based on the high axial resolution $(\sim 1 \mu \mathrm{m})$, this technique provides the capability to reveal various $3 \mathrm{D}$ transient structures without disrupting the development process. Since no extrinsic or intrinsic fluorescence was used, misgivings of abnormal development due to external disturbance do not have to be concerned as the results of no phototoxicity and photobleaching.

By comparing with the previous time-lapse study of the zebrafish embryo development, the dynamic process of brain 


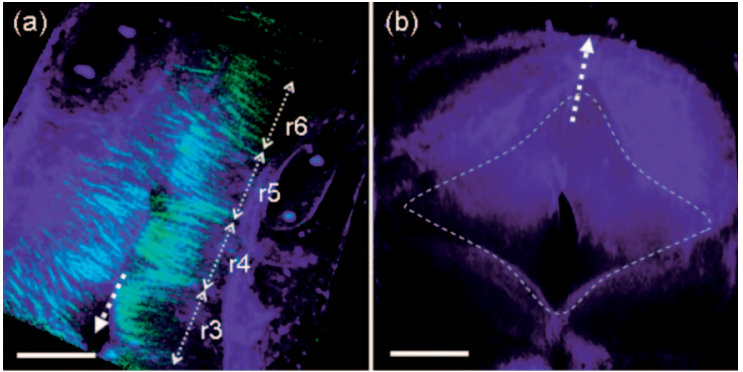

Fig. 4 Reconstructed 3D HOM images of the (a) hindbrain of a prim12-stage and (b) midbrain of a 25-somite-stage zebrafish embryo. The dotted arrow shows the posterior-to-anterior direction. In (a), 3D structures of the neural tube and optic vesicles are revealed by THG, while SHG shows the 3D neural network in the neural tube. From THG, four sections of rhombomere, r3 (bottom)-r6 (top), are shown in this image. THG in (b) shows the cavity in the midbrain (dotted line), while no SHG is shown in (b). Scale bar: $50 \mu \mathrm{m}$. THG and SHG are denoted by blue and green colors, respectively.

development we recorded matched the previous reported results completely. This identity indicates that our reported technique induced no observable biodamages so that there is no reduction in cloning efficiency and each cell could function and divide normally to accomplish the whole development process.

\subsection{SHG Signals in the Neural Tube}

During the whole process of the brain development, interfacesensitive THG provides the 3D sketch of different structures from a neural plate to a hollow neural tube. On the other hand, nerve-fiber- and mitotic-spindle-sensitive SHG can tell us much more stories about how cells behave during the development process, as in Fig. 2. When a neural plate turns into a neural keel, although most cells have differentiated into functioning cells, there is still a small group of cells that have retained the capability of mitosis. Those cells are the so-called stem cells or precursor cells. Take an image of the zebrafish hindbrain, for example. Figure 3(a) shows a sectioned HOM image of the hindbrain and the otic vesicles obtained from a live prim-5-stage zebrafish embryo. If we enlarged the small area circumscribed by the white square [Fig. 3(b)], two different sources of SHG signals can be identified to be the mitotic spindles (arrow) and the nerve fibers (arrowhead). It is interesting to note that each SHG source has its specific spatial distribution, and the distribution changes with development stages. Through the dynamic tracking of the mitosis process and the polarized neuron formation, the neural stem cell behaviors can thus be revealed without the help of fluorescence markers.

Once the neural keel begins to form, cell cycle occurring in it will go along with migration of the nuclei. ${ }^{44,46}$ According to Kimmel et al.,4 "During the cell cycle, all the cells of the germinal epithelium are continuous from the luminal surface of the neural tube to the outside surface. The nuclei move within their cells as they go through the cell cycle and mitotic cells can only be found near the luminal surface." Following the distributions and the transient behaviors of the observed SHG signals, we can easily divide the neural tube longitudinally into three distinguishable parts in order from the middle

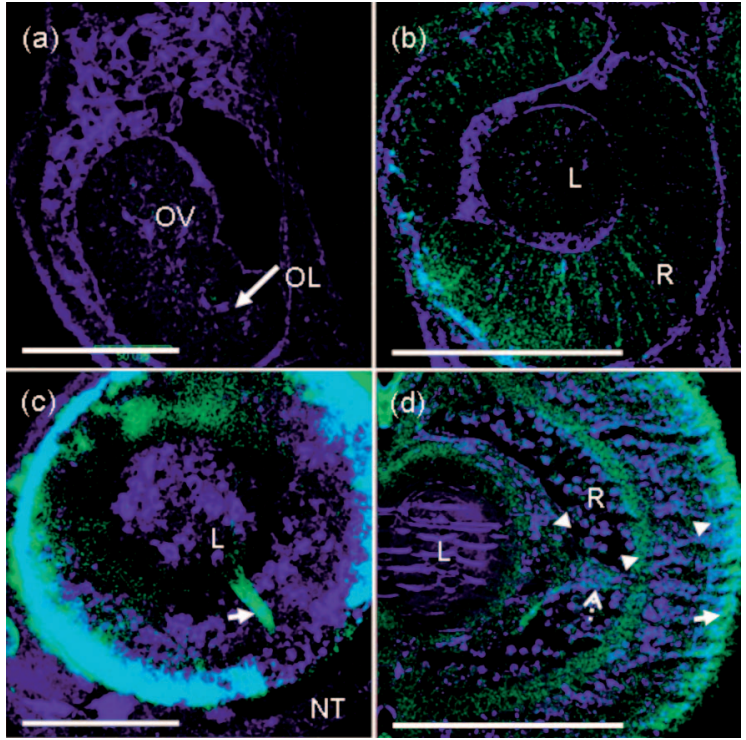

Fig. 5 HOM images of the zebrafish eye. (a) The left eye of a live 14-somite-stage embryo with the left side to the top. The flat tissue named optic vesicle (OV) and the silt, optic lumen (OL, arrow), were shown by THG. At this stage, only few SHG from mitotic spindles could be found near the optic lumen. (b) The left eye of a live prim15-stage embryo. The lens (L) and retina (R) were clearly observed through THG, and the nerve fibers elongated across the retina were picked up by SHG, respectively. (c) The eye of a live 60-hpf embryo. By superimposing each section at different depths, the optic nerve from the lens (L) to the neural tube (NT) was revealed by SHG (arrow). (d) HOM image obtained from a paraffin section of a 4-day eye. Strong SHG could be found in layers full of nerve fibers (arrowhead), the optic nerve (dotted arrow), and the outer segment of the photoreceptor (arrow). Scale bar: $100 \mu \mathrm{m}$. THG and SHG are denoted by blue and green colors, respectively.

line to the out wall. Figure 3(a) shows SHG signals highly concentrated in the region near the middle line, while the other region close to the outer wall has only a few weak SHG signals. The latter is the marginal zone; because of the lighter color of the glia cells, it is also called "white matter." Moreover, from the enlarged image [Fig. 3(b)], SHG from cell mitosis (arrow) can only be found in the ventricular zone, a narrow region close to the lumen. Zooming in again, we could see more clearly the cells [THG in Fig. 3(c)] and the mitotic spindles [SHG in Fig. 3(c); arrow]. Besides the ventricular zone, the part between the ventricular and marginal zone is the mantle zone, the so-called gray matter. According to a previous study, mitosis will only occur in the ventricular zone, and differentiated cells will leave this region to become glia or ganglia cells. ${ }^{46}$ While glia cells distribute mostly over the marginal zone, ganglia cells will elongate their nerve fibers into both mantle and marginal zones in the direction perpendicular to the neural tube. ${ }^{46}$ Thus, strong SHG signals arising from the nerve fibers [arrowhead in Fig. 3(b)] can be used to record the development and elongation of the neurons. Through long-term monitoring of the ventricular zone, the above-mentioned cell division and differentiation behaviors were all successfully tracked in vivo with HOM. Moreover, this cell cycle not only occurs in the brain but also takes place in other organs, such as the eyes. Moving our focus to the outer layer of the retina, called the optic lumen, similar to the 
brain development, SHG signals from cell mitosis could also be found [arrow in Fig. 3(d)]. By in vivo tracking of the SHG signals, we have also successfully identified the regions where the cell cycle took place without using any dye or fluorescence technique.

\subsection{D Reconstructed Image of the Neural Tube}

Due to the nonlinearity of the higher-order harmonic generations, localized excitation and submicron lateral resolution (with $1-\mu \mathrm{m}$ axial resolution) can all be achieved even with a 0.9 NA objective. Thus, HOM provides the excellent optical sectioning power inside a live embryo to reveal its $3 \mathrm{D}$ microstructures. We put a prim-5-stage live zebrafish embryo dorsal to the top and moved the scanning focal plane from the dorsal to the ventral side with a $1-\mu \mathrm{m}$ axial step between adjacent $2 \mathrm{D}$ sections. A series of THG images (shown by blue color) obtained from different depths can be reconstructed to show the 3D microstructures, for example, the hindbrain and the otic vesicles [Fig. 4(a)]. From THG, four sections of rhombomere, r3 (bottom)-r6 (top), were shown in this image. ${ }^{47}$ SHG from nerve fibers can be reconstructed to reveal the $3 \mathrm{D}$ neural network inside the neural tube. Moving the scanning plane to the midbrain of a 25 -somite-stage live embryo and collecting only the THG signals, we can clearly observe the morphological structures around the cavity (marked with the dotted line) from the process of cavitation. Because no markers and no physical sectioning were used, 3D structures can be revealed without any misgivings resulting from direct or indirect damages to the embryo. Combined with the leastinvasive nature of the demonstrated technique, it is no longer difficult to study in vivo the 3D morphology of the whole brain development on a long-term basis.

\subsection{In Vivo Observation of Eye Development}

From the results shown above, long-term in vivo observation and the 3D imaging of the brain development were carried out on the basis of the least-invasive nature, highly penetrative capability, and submicron lateral resolution provided by HOM. Combined with SHG arising from mitotic spindles and nerve fibers, the eye, an important part of the peripheral nervous system, could also be studied. Due to the simply characterized and similar structure of zebrafish eyes corresponding to human eyes, the zebrafish offers a good model for understanding the eye diseases.

Development of eyes is known to be later than that of brain. From the image shown in Fig. 2(d), the primordium of eyes besides the neural rod could be observed at the 14-somite stage. Due to the fact that the observed embryo was free to move without any spatial constraint, the observation in one specific embryo was always limited to some specific angles that we cannot control. By using another 14-somite-stage live embryo, which is different from that shown in Fig. 2, and setting it with the left side upward during the observation, a flat tissue with a slit in the middle was revealed through THG signals [Fig. 5(a)]; only few SHG could be found near the slit [SHG may be too weak to be identified in Fig. 5(a)]. When we enlarged the sectioned HOM image, it was interesting to see that SHG originated from mitotic spindles. According to the study of eye development, ${ }^{48}$ this tissue is called the optic vesicle and the slit is the optic lumen, which has been men- tioned in the discussion of Fig. 3(d). By continuously monitoring the development process in the same embryo, this flat tissue could be found to transform into the shape of a cup, called the eye cup, at the prim-5 stage, and the lens could also be distinguished then. Just after another $4-5 \mathrm{hr}$ at the prim-12 to the prim-14 stage, little SHG was found at the bottom of the eye cup, which was then called the retina. After the prim-14 stage, the observed SHG gradually became denser and stronger in the retina. It was shown that the first neuron in the retina was born and begins to elongate its nerve fibers at about the prim-12 stage to the prim-14 stage, ${ }^{48}$ at which we found SHG signals in the retina. Figure 5(b) shows an optical section of the left eye of the live prim-15-stage zebrafish embryo. The outline of the lens and the retina was shown distinctly by the THG modality, while the SHG revealed the nerve fibers in the early developed retina.

By observing in vivo another live 60-hpf (the embryo was kept at $28^{\circ} \mathrm{C}$ before observation) embryo with its left side upward and optically sectioning the eyes downward from the lens to the retina and then the neural tube, SHG from optic nerves was observed from the lens to the neural tube. Figure 5(c) shows the superimposed image of images obtained from different depths. In addition to the optical nerves [arrow in Fig. 5(c)], strong signals in the SHG channel were also found at the bottom of the retina. Most interesting was that different from the results in Dombeck et al., ${ }^{40} \mathrm{SHG}$ also arose from the optical nerve [arrow in Fig. 5(c)], which is known to consist of nerve fibers with the myelin sheath. In order to further identify the source of SHG at the bottom of the retina, we used a paraffin section of an eye of a 4-day (the embryo was kept at $28^{\circ} \mathrm{C}$ before sectioning) zebrafish embryo [Fig. 5(d)]. As expected, SHG signals had good contrast in every layer full of nerve fibers, including inner and outer plexform layers [arrowhead in Fig. 5(d)] and optical nerve [dotted arrow in Fig. 5(d)]. Besides the layer full of nerve fibers, the source of strong SHG signals was found to be the outer segment of the photoreceptors [arrow in Fig. 5(d)]. As the same origin for the SHG in grana, ${ }^{15}$ the strong SHG observed in the outer segment was the result of the stacked membranes.

According to different characteristics, SHG signals carry much information about how cells are behaving (for example, the cell mitosis process and the construction of nerve fibers), while THG shows us the morphological changes during the development process. Combined with the capability for longterm in vivo observation, HOM offers an excellent tool to study eye development. Many studies, such as the repair of the optic nerve and the disease-causing genes of eyes, can be carried out by observing the repairing process and the morphological changes resulting from the mutation of the diseasecausing genes.

\section{Conclusion}

In this work, by utilizing the endogenous SHG and THG signals, we have successfully observed in vivo the complete vertebrate embryonic nervous development from the very beginning with a submicron lateral resolution, for the first time. The SHG contrasts were found to be provided by the polarized nerve fibers, either myelinated or unmyelinated, the mitosis spindles, and the outer segments of the photoreceptors. THG was used to reveal 3D morphological changes. The complete 
and dynamic 3D development of the nervous system within a live zebrafish embryo was successfully recorded continuously for more than $20 \mathrm{hr}$ without any fluorescence markers. Since the THG process is not limited by the time of gene expression and differentiation as fluorescence-based techniques are, the observable stages can be advanced to the very beginning of the whole development process, at least $14 \mathrm{hr}$ sooner than the fluorescence-based technique. The complete 3D brain development from a neural plate to a neural tube can be uncovered without any treatment on the studied live specimens. Since no fluorophores and live markers are needed, toxicity, photobleaching, and other biological as well as genetic environment modifications can all be completely avoided. After observing the brain development of several tens of zebrafish embryos, with nonstop, continuous observation time in a single embryo, even up to $20 \mathrm{hr}$ the embryos can all develop normally into the larva stages. We have, for the first time, also reported the generation of SHG from myelinated nerve fibers and from the outer segment of the photoreceptors with a stacked membrane structure. Furthermore, with the help of SHG-signaled mitosis process, we showed the possibility to track neural stem cells without using fluorescence markers. Our study clearly indicates the fact that higher-harmonicsbased optical microscopy has the strong potential to long-term in vivo study of the nervous system, including genetic disorders of the nervous system, axon pathfinding, neural regeneration, neural repair, and neural stem cell development.

\section{Acknowledgments}

The authors would like to acknowledge the support by the National Health Research Institute through Grant NHRIEX95-9201EI and the NTU Center for Genomic Medicine of Taiwan.

\section{References}

1. C.-K. Sun, "Higher harmonic generation microscopy," Adv. Biochem. Eng./Biotechnol. 95, 17-56 (2005).

2. R. W. Boyd, Nonlinear Optics, 2nd ed., Academic Press, Boston, Mass. (1992).

3. D. Yelin and Y. Silberberg, "Laser scanning third-harmonicgeneration microscopy in biology," Opt. Express 5, 169-175 (1999).

4. D. Oron, D. Yelin, E. Tal, S. Raz, R. Fachima, and Y. Silberberg, "Depth-resolved structural imaging by third-harmonic generation microscopy," J. Struct. Biol. 147, 3-11 (2004).

5. T. Y. F. Tsang, "Optical 3rd-harmonic generation at interfaces," Phys. Rev. A 52, 4116-4125 (1995).

6. J. A. Squier, M. Muller, G. J. Brakenhoff, and K. R. Wilson, "Third harmonic generation microscopy," Opt. Express 3, 315-324 (1998).

7. Y. Barad, H. Eisenberg, M. Horowitz, and Y. Silberberg, "Nonlinear scanning laser microscopy by third harmonic generation," Appl. Phys. Lett. 70, 922-924 (1997).

8. M. Muller, J. Squier, K. R. Wilson, and G. J. Brakenhoff, "3D microscopy of transparent objects using third-harmonic generation," $J$. Microsc. 191, 266-274 (1998).

9. S.-W. Chu, S.-P. Tai, C.-L. Ho, C.-H. Lin, and C.-K. Sun, "Highresolution simultaneous three-photon fluorescence and thirdharmonic-generation microscopy," Microsc. Res. Tech. 66, 193-197 (2005).

10. V. Barzda, "Visualization of mitochondria in cardiomyocytes by simultaneous harmonic generation and fluorescence microscopy," Opt. Express 13, 8263-8276 (2005).

11. A. C. Millard, D. N. Fittinghoff, P. W. Wiseman, M. Muller, G. J. Brakenhoff, J. A. Squier, and K. R. Wilson, "Three dimensional, third harmonic microscopy of living systems," Biophys. J. 78, 800Plat Part 2 (2000).
12. D. Debarre, W. Supatto, and E. Beaurepaire, "Structure sensitivity in third-harmonic generation microscopy," Opt. Express 30, 2134-2136 (2005).

13. S.-W. Chu, S.-Y. Chen, T.-H. Tsai, T.-M. Liu, C.-Y. Lin, H.-J. Tsai, and C.-K. Sun, "In vivo developmental biology study using noninvasive multi-harmonic generation microscopy," Opt. Express 11, 3093 3099 (2003)

14. C.-K. Sun, S.-W. Chu, S.-Y. Chen, T.-H. Tsai, T.-M. Liu, C.-Y. Lin, and H.-J. Tsai, "Higher harmonic generation microscopy for developmental biology," J. Struct. Biol. 147, 19-30 (2004).

15. S.-W. Chu, I.-H. Chen, T.-M. Liu, C.-K. Sun, S.-P. Lee, B.-L. Lin, P. C. Cheng, M.-X. Kuo, D.-J. Lin, and H.-L. Liu, "Nonlinear biophotonic crystal effects revealed with multimodal nonlinear microscopy," J. Microsc. 208, 190-200 Part 3 (2002).

16. J. Y. Huang, Z. P. Chen, and A. Lewis, "2nd-harmonic generation in purple membrane-poly(vinyl alcohol) films-probing the dipolar characteristics of the bacteriorhodopsin chromphore in br570 and m412," J. Phys. Chem. 93, 3314-3320 (1989).

17. W. Mohler, A. C. Millard, and P. J. Campagnola, "Second harmonic generation imaging of endogenous structural proteins," Methods 29, 97-109 (2003).

18. S.-W. Chu, I.-H. Chen, T.-M. Liu, P. C. Cheng, and C.-K. Sun, "Multimodal nonlinear spectral microscopy based on a femtosecond Cr:forsterite laser," Opt. Lett. 26, 1909-1911 (2001).

19. P. J. Campagnola, A. C. Millard, M. Terasaki, P. E. Hoppe, C. J. Malone, and W. A. Mohler, "Three-dimensional high-resolution second-harmonic generation imaging of endogenous structural proteins in biological tissues," Biophys. J. 82, 493-508 (2002).

20. P. J. Campagnola and L. M. Loew, "Second-harmonic imaging microscopy for visualizing biomolecular arrays in cells, tissues and organisms," Nat. Biotechnol. 21, 1356-1360 (2003).

21. S.-W. Chu, S.-Y. Chen, G.-W. Chern, T.-H. Tsai, Y.-C. Chen, B.-L. Lin, and C.-K. Sun, "Studies of $\chi^{(2)} / \chi^{(3)}$ tensors in submicron-scaled bio-tissues by polarization harmonics optical microscopy," Biophys. J. 86, 3914-3922 (2004).

22. R. M. Williams, W. R. Zipfel, and W. W. Webb, "Interpreting secondharmonic generation images of collagen I fibrils," Biophys. J. 88, 1377-1386G (2005).

23. G. Cox, E. Kable, A. Jones, I. K. Fraser, F. Manconi, and M. D. Gorrell, "3-dimensional imaging of collagen using second harmonic generation," J. Struct. Biol. 141, 53-62 (2003).

24. A. F. Schier, S. C. Neuhauss, M. Harvey, J. Malicki, L. SolnicaKrezel, D. Y. R. Stainier, F. Zwartkruis, S. Abdelilah, D. L. Stemple, Z. Rangini, H. Yang, and W. Driever, "Mutations affecting the development of the embryonic zebrafish brain," Development 123, 165-178 (1996).

25. F. S. Foster, C. J. Pavlin, K. A. Harasiewicz, D. A. Christopher, and D. H. Turnbull, "Advances in ultrasound biomicroscopy," Ultrasound Med. Biol. 26, 1-27 (2000).

26. Y. Z. Wadghiri, J. A. Blind, X. H. Duan, C. Moreno, X. Yu, A. L. Joyner, and D. H. Turnbull, "Manganese-enhanced magnetic resonance imaging (MEMRI) of mouse brain development," NMR Biomed. 17, 613-619 (2004).

27. M. Modo, K. Mellodew, D. Cash, S. E. Fraser, T. J. Meade, J. Price, and S. C. R. Williams, "Serial in vivo magnetic resonace imaging of stroke-induced transhemispheric neural stem cell migration," Exp. Neurol. 181, 100-100 (2003).

28. S. Yazdanfar, A. M. Rollins, and J. A. Izatt, "In vivo imaging of human retinal flow dynamics by color Doppler optical coherence tomography," Arch. Ophthalmol. (Chicago) 121, 235-239 (2003).

29. T. M. Yelbuz, M. A. Choma, L. Thrane, M. L. Kirby, and J. A. Izatt, "Optical coherence tomography-a new high-resolution imaging technology to study cardiac development in chick embryos," Circulation 106, 2771-2774 (2002).

30. C. Palmes-Saloma and C. Saloma, "Long-depth imaging of specific gene expressions in whole-mount mouse embryos with single-photon excitation confocal fluorescence microscopy and FISH," J. Struct. Biol. 131, 56-66 (2002).

31. J. C. Kasemeier-Kulesa, P. M. Kulesa, and F. Lefcort, "Imaging neural crest cell dynamics during formation of dorsal root ganglia and sympathetic ganglia," Development 132, 235-245 (2005).

32. A. D. Chalmers, B. Strauss, and N. Papalopulu, "Oriented cell divisions asymmetrically segregate aPKC and generate cell fate diversity in the early Xenopus embryo," Development 130, 2657-2668 (2003) 
33. E. S. Ruthazer and H. T. Cline, "Multiphoton imaging of neurons in living tissue: Acquisition and analysis of time-lapse morphological data," Real-Time Imag. 8, 175-188 (2002).

34. M. J. Levene, D. A. Dombeck, K. A. Kasischke, R. P. Molloy, and W. W. Webb, "In vivo multiphoton microscopy of deep brain tissue," J. Neurophysiol. 91, 1908-1912 (2004).

35. E. Brustein, N. Marandi, Y. Kovalchuk, P. Drapeau, and A. Konnerth, "In vivo monitoring of neuronal network activity in zebrafish by twophoton $\mathrm{Ca}^{2+}$ imaging," Pfluegers Arch. 446, 766-773 (2003).

36. C. Stosiek, O. Garaschuk, K. Holthoff, and A. Konnerth, "In vivo two-photon calcium imaging of neuronal networks," Proc. Natl. Acad. Sci. U.S.A. 100, 7319-7324 (2003).

37. J. Liu, S. A. Ernst, S. E. Gladycheva, Y. Y. Lee, S. I. Lentz, C. S. Ho, Q. Li, and E. L. Stuenkel, "Fluorescence resonance energy transfer reports properties of syntaxin1a interaction with Munc18-1 in vivo," Biol. Chem. 279, 55924-55936 (2004).

38. J. N. Post, K. A. Lidke, B. Rieger, and D. J. Arndt-Jovin, "One- and two-photon photoactivation of a paGFP-fusion protein in live Drosophila embryos," FEBS Lett. 579, 325-330 (2005).

39. K. Konig, "Multiphoton microscopy in life sciences," J. Microsc. 200, 83-104 (2000)

40. D. A. Dombeck, K. A. Kasischke, H. D. Vishwasrao, M. Ingelsson, B. T. Hyman, and W. W. Webb, "Uniform polarity microtubule assemblies imaged in native brain tissue by second-harmonic generation microscopy," Proc. Natl. Acad. Sci. U.S.A. 100, 7081-7086
(2003).

41. D. A. Dombeck, M. Blanchard-Desce, and W. W. Webb, "Optical recording of neuronal action potentials with second-harmonic generation microscopy," Biophys. J. 86, 317A-317A Part 2 (2004).

42. S.-P. Tai, T.-H. Tsai, W.-J. Lee, D.-B. Shieh, Y.-H. Liao, H.-Y. Huang, K.Y.-J. Zhang, H.-L. Liu, and C.-K. Sun, "Optical biopsy of fixed human skin with backward-collected optical harmonics signals," Opt. Express 13, 8231-8242 (2005).

43. N. Milos and A. D. Dingle, "Dynamics of pigment pattern formation in the zebrafish, Brachydanio rerio. (1) Establishment and regulation of the lateral line melanophore stripe during the first eight days of development," J. Exp. Zool. 205, 205-216 (1978).

44. C. B. Kimmel, W. W. Ballard, S. R. Kimmel, B. Ullmann, and T. F. Schilling, "Stages of embryonic development of the zebrafish," Dev. Dyn. 203, 253-310 (1995).

45. G. B. Downes, J. A. Waterbury, and M. Granato, "Rapid in vivo labeling of identified zebrafish neurons," Genesis 34, 196-202 (2002).

46. C. B. Moens and V. E. Prince, "Constructing the hindbrain: Insights from the zebrafish," Dev. Dyn. 224, 1-17 (2002).

47. S. F. Gilbert, Developmental Biology, 7th ed., Sinauer Associates, Sunderland, Mass. (2003).

48. Z. Li, N. M. Joseph, and S. S. Easter, Jr., "The morphogenesis of the zebrafish eye, including a fate map of the optic vesicle," Dev. Dyn. 218, 175-188 (2000). 